\title{
Psychological distress following a motor vehicle crash: preliminary results of a randomised controlled trial investigating brief psychological interventions
}

\author{
Rebecca Guest ${ }^{*}$ D, Yvonne Tran, Bamini Gopinath, Ian D. Cameron and Ashley Craig
}

\begin{abstract}
Background: The preliminary results of a randomised controlled trial are presented. The aim of the trial is to determine the efficacy, feasibility and acceptability of email-delivered psychological interventions with telephone support, for adults injured in a motor vehicle crash engaged in seeking compensation. The primary intention for this preliminary analysis was to investigate those who were psychologically distressed and to stop the trial midway to evaluate whether the safety endpoints were necessary.

Methods: The analysis included 90 adult participants randomised to one of three groups, who were assessed at baseline and post-intervention at 3 months. Cognitive behaviour therapy (CBT) or healthy lifestyle interventions were delivered over 10 weeks, involving fortnightly emailed modules plus clinically focussed telephone support, with the aim of reducing psychological distress. An active waiting list of control subjects received non-clinically focussed telephone contact over the same period along with claim-related reading material. Depression Anxiety Stress Scales (DASS) and Impact of Events Scale (Revised) (IES-R) were used to assess psychological distress. Psychiatric interviews were used to diagnose major depressive disorder and post-traumatic stress disorder. Aspects of acceptability and feasibility were also assessed.
\end{abstract}

Results: For those diagnosed with depression at baseline in the CBT group, psychological distress reduced by around $16 \%$. For those with depression in the healthy lifestyle group, distress increased marginally. For those in the control group with depression, distress also decreased (by 18\% according to DASS-21 and 1.2\% according to IES-R). For those without depression, significant reductions in distress occurred, regardless of group $(P<.05)$. The results suggest that for those with depression, a healthy lifestyle intervention is contraindicated, necessitating the cessation of recruitment to this intervention. The interventions were reported as acceptable by the majority and the data indicated that the study is feasible.

Conclusions: CBT with telephone support reduced psychological distress in physically injured people with depression who are engaged in seeking compensation. However, time plus fortnightly telephone contact with claim-related reading material may be sufficient to reduce distress in those who are depressed. For those who were not depressed, time plus telephone support is most likely sufficient enough to assist them to recover. The trial will continue with further recruitment to only the CBT and control groups, over longer follow-up periods.

(Continued on next page)

\footnotetext{
* Correspondence: rebecca.guest@sydney.edu.au

John Walsh Centre for Rehabilitation Research, Kolling Institute of Medical

Research, Sydney Medical School-Northern, The University of Sydney, Corner

Reserve Road and First Avenue, Royal North Shore Hospital, St Leonards,

NSW 2065, Australia
}

(c) The Author(s). 2018 Open Access This article is distributed under the terms of the Creative Commons Attribution 4.0 International License (http://creativecommons.org/licenses/by/4.0/), which permits unrestricted use, distribution, and reproduction in any medium, provided you give appropriate credit to the original author(s) and the source, provide a link to the Creative Commons license, and indicate if changes were made. The Creative Commons Public Domain Dedication waiver (http://creativecommons.org/publicdomain/zero/1.0/) applies to the data made available in this article, unless otherwise stated. 
(Continued from previous page)

Trial registration: Australian and New Zealand Clinical Trials Registry: Preventing psychological distress following a motor vehicle accident; ACTRN12615000326594. Registered on 9 April 2015.

Keywords: CBT/cognitive behaviour therapy, Depression, Anxiety, PTSD/post-traumatic stress disorder, MVC/motor vehicle crash, Injury

\section{Background}

Psychological distress is elevated in those who have experienced a motor vehicle crash (MVC) [1-5]. The psychosocial impacts of non-catastrophic injuries sustained in MVCs can be debilitating and include chronic pain, disability, loss of income, trauma and stress placed on relationships $[5,6]$. The risk of a psychological disorder, such as major depressive disorder (MDD) and post-traumatic stress disorder (PTSD), is also high [1, 7]. Furthermore, involvement in seeking MVC-related compensation is associated with increased levels of psychological distress compared to those not claiming [8]. Research has shown that psychological distress and rates of MDD and PTSD remain elevated up to at least 12 months post-MVC [5, 9].

Given the above, a strategy for reducing the risk of psychological distress would involve providing brief interventions to MVC survivors engaged in seeking compensation as soon after the MVC as practicable [10-12]. Potential interventions include cognitive behaviour therapy (CBT) involving, for example, psychoeducation about the dynamics of distress and injury, stress reduction and helpful thinking techniques, the goal being to enhance adaptive psychological, social and behavioural skills. Another strategy involves healthy lifestyle (HL) interventions focussing on sleep, diet and exercise [13-15].

CBT is an efficacious treatment for disorders such as MDD and PTSD [11, 15-19]. Likewise, HL interventions have been shown to increase well-being $[14,15,20]$, and there is growing support for improvements in mental health associated with increased regular physical activity and improved sleep [13, 21-25].

The internet provides a novel platform for the delivery of psychological interventions, by way of an online biblio-therapy with therapist contact $[18,26]$. Evidence exists for the efficacy of this style of delivery for lifestyle and psychological disorders [14, 27]. Further, a systematic review identified that an online-delivered CBT program was as effective as face-to-face CBT in reducing depressive symptoms $[18,28,29]$. Email delivery has the advantage of no web-based costs, and arguably results in personable delivery of CBT, especially if clinically focussed telephone support is provided. If the trial shows the benefit of such brief interventions, internet-based modes of delivery could be explored.
Given the very limited research into the management of psychological distress in MVC compensation claimants [11], there is a need for controlled research into the efficacy of strategies designed to lower risk of distress and disorder in this area. Therefore, adults who experienced a non-catastrophic injury in an MVC and were engaged in seeking compensation were invited to participate this study. The goal was to determine the efficacy of CBT and HL interventions, integrated with clinically focussed telephone support, to reduce psychological distress compared to a control group of people with an MVC injury engaged in seeking compensation. Whilst it is expected that the majority of people will recover regardless of the intervention [5], we believe that those who have elevated psychological distress at baseline will be more complex to treat and require careful monitoring. Given the vulnerability of those with elevated psychological distress, the primary intention of the preliminary analysis was to compare those with a baseline diagnosis of MDD versus those without a diagnosis of MDD. A further primary intention was to determine the necessity of implementing the safety endpoints for either of the interventions, should the results show any degree of harm. It was hypothesised that those without MDD would show improvement at the 3-month assessment, with the CBT and HL groups showing superior improvement. It was also hypothesised that those with MDD in the CBT and HL groups would show significantly reduced distress compared to the control group.

\section{Methods \\ Participants}

The details of the protocol, such as recruitment, design, setting and aims for this randomised controlled trial (RCT), have been previously reported in the study's published protocol paper [30]. A CONSORT checklist file shows how the recommendations for a clinical trial have been addressed (Additional file 1). Participants included MVC survivors who lodged a compensation claim in New South Wales (NSW) or Victoria, Australia, between July 2015 and May 2017. Subsequent to publication of the protocol paper [30], a third recruitment site was secured in NSW. Recruitment sites included: (1) Suncorp and (2) the National Roads and Motorists' Association in 
NSW and (3) the Transport Accident Commission in Victoria. Figure 1 shows the flow for the study's recruitment, intervention and assessment processes. The NSW compulsory third-party insurance system is fault based; therefore, none of the NSW people in the study were at fault in their crash, whereas Victoria participants could be either at fault or not at fault. The inclusion criteria were (1) adult (age > 18 years) survivor of an MVC who lodged a claim within 4 months of their MVC and (2) English speaking. Exclusion criteria included the presence of severe injuries, such as spinal cord injury, amputation, blindness, severe traumatic brain injury and other injuries requiring extended hospitalisation. Table 1 shows participant characteristics including socio-demographic, injury and intervention-related characteristics by group.

\section{Design}

This RCT is ongoing, with participants being assessed across four time points: baseline assessment upon entry into the RCT (mean 12 weeks post-MVC), and 3 , 6, and 12 months post-intervention. For this preliminary investigation, the trial was stopped at the midway point when 30 participants in each group had completed the baseline, the intervention and the 3 -month post-intervention assessment.

\section{Sample size}

Based on prior results for the CBT and HL interventions $[7,14,15]$, the two interventions were assumed to have at least a small to moderate mean effect size of 0.25 (Cohen's $d$ ) compared to the control group. Assuming $\alpha=0.05$ and a power of $80 \%$ for a three-group comparison analysis with four measures over time, a sample of 135 participants needed to be recruited to detect true differences [30]. It was, therefore, proposed to recruit at least 180 participants to accommodate loss to follow-up of approximately $25 \%$ based on similar research [31].

\section{Procedure}

The preliminary analysis shows a total of 240 individuals received information about the trial, of whom 104 were randomised into one of three groups: CBT $(n=37)$, HL $(n=35)$ or active waiting list (control, $n=32$ ). We included 104 participants in this analysis to ensure attrition did not impact on the 30/30/30 preliminary analysis. The CBT and HL groups received fortnightly

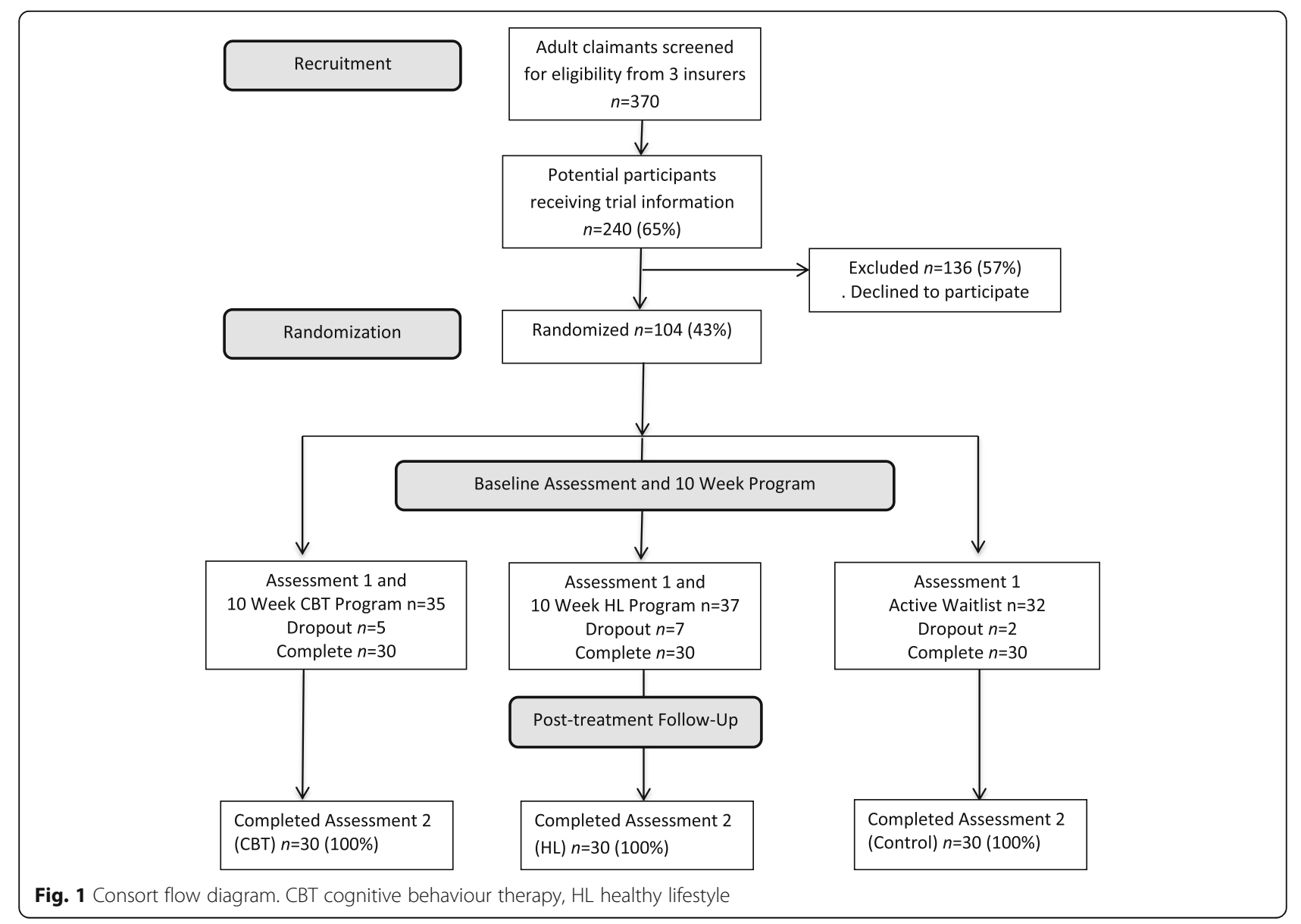


Table 1 Participant characteristics by group including demographic, injury and intervention-related characteristics

\begin{tabular}{|c|c|c|c|c|}
\hline Characteristics & Control & $\mathrm{HL}$ & CBT & Total \\
\hline Age: mean years (SD) & $47.67(14.8)$ & $47.83(13.2)$ & $42.70(16.0)$ & $46.07(14.7)$ \\
\hline Female $n(\%)$ & $21(70.0)$ & $19(63.3)$ & $21(70.0)$ & $61(67.8)$ \\
\hline $\mathrm{BMI} M(\mathrm{SD})^{\mathrm{a}}$ & $26.31(5.5)$ & $29.74(6.0)$ & 28.90(9.1) & $28.27(7.2)$ \\
\hline \multicolumn{5}{|l|}{ Marital status $n(\%)$} \\
\hline Single & $5(16.7)$ & $4(13.3)$ & $8(26.7)$ & 17(18.9) \\
\hline Widowed & $0(0.0)$ & $1(3.3)$ & $2(6.7)$ & $3(3.3)$ \\
\hline Divorced/separated & $7(23.3)$ & $3(10.0)$ & $9(30.0)$ & 19(21.1) \\
\hline Married/de facto & 18(60.0) & $22(73.4)$ & 11(36.6) & $51(56.7)$ \\
\hline \multicolumn{5}{|l|}{ Education $n(\%)$} \\
\hline Up to year 10 & $8(26.7)$ & $5(16.7)$ & $5(16.7)$ & 18(20.0) \\
\hline Year 12 or equivalent & $3(10.0)$ & $4(13.3)$ & $3(10.0)$ & 10(11.1) \\
\hline Technical or further education & $9(30.0)$ & $3(10.0)$ & $7(23.3)$ & 19(21.1) \\
\hline University & 10(33.3) & 18(60.0) & $15(50.0)$ & $43(47.8)$ \\
\hline \multicolumn{5}{|l|}{ Work $n(\%)$} \\
\hline Full time & 16(53.3) & $14(46.7)$ & $17(56.7)$ & $47(52.2)$ \\
\hline Part time & $8(26.8)$ & $8(26.7)$ & $7(23.3)$ & 23(25.6) \\
\hline Pensioner & $4(13.3)$ & $7(23.3)$ & $4(13.3)$ & 15(16.7) \\
\hline Unemployed & $1(3.3)$ & $1(3.3)$ & $2(6.7)$ & $4(4.4)$ \\
\hline Student & $1(3.3)$ & $0(0.0)$ & $0(0.0)$ & $1(1.1)$ \\
\hline \multicolumn{5}{|l|}{ Role in MVC } \\
\hline Driver & 13(43.3) & $21(70.0)$ & $18(60.00)$ & $52(57.8)$ \\
\hline Passenger & $5(16.7)$ & $6(20.0)$ & $2(6.66)$ & 13(14.4) \\
\hline Motorbike rider & $5(16.7)$ & $0(0.0)$ & $8(26.66)$ & 13(14.4) \\
\hline Bicyclist & $3(10.0)$ & $3(10.0)$ & $1(3.33)$ & $7(7.8)$ \\
\hline Pedestrian & $4(13.3)$ & $0(0.0)$ & $1(3.33)$ & $5(5.6)$ \\
\hline Days since MVC: M (SD) & $96.77(71.8)$ & $81.13(52.3)$ & $68.37(41.2)$ & $82.09(57.1)$ \\
\hline Days in hospital: $M(S D)$ & $0.80(1.7)$ & $1.37(4.2)$ & $0.93(1.8)$ & $1.03(2.8)$ \\
\hline \multicolumn{5}{|l|}{ Injury type/location } \\
\hline Neck $n(\%)$ & $5(17.2)$ & 11(36.8) & $8(28.6)$ & $24(27.6)$ \\
\hline Shoulder $n(\%)$ & $4(13.8)$ & $1(3.3)$ & $2(7.1)$ & $7(8.1)$ \\
\hline $\operatorname{Arm} n(\%)$ & $2(6.9)$ & $1(3.3)$ & $2(7.1)$ & $5(5.7)$ \\
\hline Upper back $n(\%)$ & $3(10.3)$ & $4(13.3)$ & $4(14.3)$ & $11(12.6)$ \\
\hline Lower back $n(\%)$ & $7(24.1)$ & $3(10.0)$ & $4(14.3)$ & 14(16.1) \\
\hline Leg $n(\%)$ & $6(20.8)$ & $6(20.0)$ & $4(14.3)$ & 16(18.4) \\
\hline Head $n(\%)$ & $0(0.0)$ & $3(10.0)$ & 1(3.6) & $4(4.6)$ \\
\hline Chest/abdomen $n(\%)$ & $2(6.9)$ & $1(3.3)$ & $3(10.7)$ & $6(6.9)$ \\
\hline Pain intensity $M(S D)^{b}$ & $7.10(1.9)$ & $6.73(2.8)$ & $6.30(2.5)$ & $6.71(2.4)$ \\
\hline Perceived danger in MVC M (SD) & $2.87(1.3)$ & $3.30(1.5)^{*}$ & $2.37(1.1)$ & $2.84(1.4)$ \\
\hline None or small: $n(\%)$ & $12(40.0)$ & $11(36.7)$ & $16(53.3)$ & $39(43.3)$ \\
\hline Moderate, great, overwhelming: $n(\%)$ & 18(60.0) & 19(63.3) & $14(46.7)$ & $51(56.7)$ \\
\hline Physical Health Composite Index: M(SD) & $33.77(7.3)$ & $34.36(10.1)$ & $36.54(9.3)$ & $34.89(9.0)$ \\
\hline Mental Health Composite Index: M(SD) & $42.09(14.0)$ & $41.74(15.4)$ & $44.79(14.3)$ & $42.88(14.5)$ \\
\hline \multicolumn{5}{|l|}{ Treated by psychologist/psychiatrist pre-MVC } \\
\hline$n$ (yes) & $6(20.00)$ & $7(23.3)$ & $15(50.0)$ & 28(31.1) \\
\hline
\end{tabular}


Table 1 Participant characteristics by group including demographic, injury and intervention-related characteristics (Continued)

\begin{tabular}{|c|c|c|c|c|}
\hline Characteristics & Control & $\mathrm{HL}$ & CBT & Total \\
\hline$n($ no) & $24(80.00)$ & 23(76.7) & 15(50.0) & $62(68.9)$ \\
\hline \multicolumn{5}{|c|}{ Psychiatric medications pre-MVC ${ }^{C}$} \\
\hline$n$ (yes) & $8(26.7)$ & $6(20.0)$ & $8(26.7)$ & $22(24.4)$ \\
\hline$n($ no) & $22(73.3)$ & $24(80.0)$ & $22(73.3)$ & 68(75.6) \\
\hline \multicolumn{5}{|c|}{ Intervention-related characteristics } \\
\hline Telephone calls $M(\mathrm{SD})^{\mathrm{d}}$ & $2.47(1.6)$ & $3.27(2.8)$ & $3.23(2.6)$ & 2.99(3.3) \\
\hline \multicolumn{5}{|l|}{ Quality of rapport $n(\%)^{\mathrm{e}}$} \\
\hline Poor & $5(16.7)$ & 7(23.33) & $2(6.7)$ & 14(15.6) \\
\hline Somewhat & $11(36.7)$ & 10(33.3) & 10(33.3) & $31(34.4)$ \\
\hline Excellent & $14(46.7)$ & 13(43.3) & 18(60.0) & $45(50.0)$ \\
\hline \multicolumn{5}{|c|}{ Email versus postal delivery $n(\%)$} \\
\hline Email & 28(93.3) & $27(90.0)$ & 29(96.7) & $84(93.3)$ \\
\hline Postal & $2(6.7)$ & $3(10.0)$ & $1(3.3)$ & $6(6.7)$ \\
\hline
\end{tabular}

$B M I$ body mass index, $C B T$ cognitive behaviour therapy, $H L$ healthy lifestyle, $M$ mean, $M V C$ motor vehicle crash, $S D$ standard deviation

${ }^{*} \mathrm{HL}$ group significantly different to CBT $(P<.05)$

${ }^{a}$ Data missing for three people for $\mathrm{HL}$; total $n=87$

bain intensity score from 1 to 10

'Medication information was obtained with the question 'Have you ever been prescribed medication for anxiety or depression?'

${ }^{\mathrm{d}}$ Telephone calls relates to the number of calls required by the researcher to achieve the participants' second assessment

${ }^{e}$ Quality of rapport between researcher and participant is a subjective rating by the researcher who made the telephone calls

modules in Microsoft PowerPoint presentation format with homework worksheets, self-monitoring templates and instructions for using CBT or HL skills. CBT and HL participants also received clinically focussed telephone support on alternate weeks to encourage and remind them to practise the skills and to read the module material. The control group received compensation-related material every second week and a phone call on the alternate week. The reading material for the control group was restricted to publicly accessible claim information, and telephone calls confirmed this information was received. Table 2 shows the content of each module for each intervention group. Preliminary findings for the first 90 participants who completed the baseline assessment, interventions and 3-month follow-up are reported here and Fig. 1 shows the flow of these participants from eligibility through to this preliminary analysis.

\section{Measures}

A selection of the measures employed in this study have been analysed for this preliminary investigation. These include socio-demographics, MVC and injury characteristics, pre-injury mental health, self-reported body mass index (BMI), pain intensity and aspects of acceptability and feasibility. Psychometric measures included the Depression, Anxiety and Stress Scale (DASS-21) and the Impact of Events Scale (Revised) (IES-R). Structured interviews based on criteria from the 5th Edition of the Diagnostic and Statistical Manual of Mental Disorders (DSM-5) were used to diagnose MDD and PTSD.

DASS-21 is a 21-item self-report scale providing an assessment of the severity of psychological distress as a total score and in three domains: depressive mood, anxiety and stress [32, 33]. Participants completed 21 4-point Likert items (0-3) assessing self-reported distress over the past week. Higher scores indicate elevated

Table 2 Contents of each of the five modules for CBT, lifestyle and waiting list control groups

\begin{tabular}{|c|c|c|c|}
\hline & CBT & Lifestyle & Control \\
\hline 1 & Overview & Overview & Reading; A guide for people injured in an MVC \\
\hline 2 & Mood and slow breathing skills, self-monitoring & Goal-setting and life-pacing & Reading: Overview of the claims process \\
\hline 3 & The art of distraction, applying distraction & $\begin{array}{l}\text { Self-monitoring and changing unhelpful } \\
\text { behaviours }\end{array}$ & Reading: Obligations of the insurer \\
\hline 4 & Stress and helpful thinking, evidenced-based thinking & Sleep hygiene and sleep self-monitoring & Reading: Obligations of the claimant \\
\hline 5 & $\begin{array}{l}\text { Problem-solving and conclusions, self-mastery, } \\
\text { changeable vs. unchangeable problems }\end{array}$ & $\begin{array}{l}\text { Improving well-being through diet and } \\
\text { physical exercise, social participation, } \\
\text { daily activity schedule }\end{array}$ & Reading: Finalising a claim \\
\hline
\end{tabular}

CBT cognitive behaviour therapy, MVC motor vehicle crash 
distress. Total scores are calculated by summing all 21 items [33], and then, in accordance with the DASS-42, scores were multiplied by 2 [33]. DASS-21 has sound psychometric properties, including acceptable internal reliability and validity [32].

IES-R is a 22-item self-report measure of trauma-related distress [34], validated in people with traffic injuries [35]. Respondents indicate their degree of distress during the past 7 days related to their recent MVC. It is a 5-point scale ranging from 0 (not at all) to 4 (extremely) with subscales for avoidance (e.g. avoidance of feelings or situations), intrusion (e.g. distressing thoughts) and hyperarousal (e.g. irritability and hypervigilance). Domains are scored by determining the mean item score [34]. Higher scores indicate increased distress.

\section{$D S M-5$}

MDD was diagnosed by clinician interview if participants met relevant DSM-5 criteria by answering the relevant questions on their online assessments. All participants had experienced trauma in their recent MVC, satisfying the first requirement for a PTSD diagnosis [36]. They also needed to report at least one of the essential PTSD symptoms described by DSM-5.

\section{Short Form 12}

The Short Form 12 (SF-12) includes 12 items from the SF-36 [37] self-report health survey. It produces the physical component summary and the mental component summary scores. Scoring of items is identical to the SF-36 and higher scores mean a higher quality of life.

\section{Acceptability and attrition}

The acceptability of the interventions was measured post-treatment for the CBT and HL groups using four questions: 0

1. Overall, how satisfied were you with the program?

2. How satisfied were you with the modules and module summaries?

3. Would you feel confident in recommending this treatment to a friend?

4. Was it worth your time doing the program?

Participants responded to the first two questions using a 5-point Likert scale, with choices ranging from 'very satisfied' to 'very dissatisfied', while the second two questions used a 'yes' or 'no' response. These questions have been used successfully in prior research to examine the acceptability of internet-delivered CBT $[38,39]$. Attrition relates to the total number of participants who dropped out prior to the second measure.

\section{Feasibility}

Feasibility was determined by analysing (1) the percentage of people offered the interventions versus those who declined, (2) the time from consent to commencement in the study, (3) the percentage attrition from randomisation through to post-treatment assessment and (4) the ease at which email delivery is available to participants by comparing the number of email-delivered versus post-delivered programs.

\section{Data analysis}

Participants were given an identification number to ensure anonymity, and an internet link to complete their assessments through Survey Gizmo, a professional online secure survey software tool. All groups completed identical assessments at baseline and post-intervention (approximately 3 months post-injury). The data analyst had no contact with participants and was blind to randomisation. The intervention program coordinator, who also provided clinically focussed telephone support, was blind to all assessment data.

The purpose of this preliminary analysis was to analyse participants diagnosed with MDD at baseline and determine whether cessation of recruitment to either intervention group was required. As part of this analysis, preliminary efficacy trends were investigated to provide some indication of acceptability and feasibility. Statistical significance at this point in the RCT analysis was not the major objective, nevertheless limited statistical analyses were performed using SPSS version 22 [40]. While it is understood these analyses were preliminary and underpowered, we believe it is important to examine efficacy trends. The analysis included descriptive statistics for the primary outcome measures (Table 3 ). General linear repeated measures MANCOVA was used to determine differences over time and between groups for the total scores from DASS-21 and IES-R. The total scores were used as primary outcomes as they provide maximum variance. As described in the original protocol [30], it was decided to adjust for variables believed to influence primary outcomes (e.g. the baseline value of a primary outcome) and those pre-specified in the protocol [41]. Therefore, as specified, physical health (the physical component summary of the baseline SF-12) was adjusted for in the DASS-21 and IES-R analyses. Further, baseline DASS-21 depressive mood was adjusted for in the DASS-21 total score analyses and IES-R intrusion was adjusted for in the IES- $\mathrm{R}$ total score analysis. These two baseline measures were chosen because they are related to the primary outcome and their inclusion as covariates that were adjusted for baseline severity improved the efficiency of the analyses. Baseline differences between groups were regarded as randomisation anomalies [42]. 
Table 3 Results for pre- and post-intervention DASS-21 and IES-R by group

\begin{tabular}{|c|c|c|c|}
\hline & Control & $\mathrm{HL}$ & CBT \\
\hline & Mean (SD) 95\% Cl & Mean (SD) 95\% Cl & Mean (SD) 95\% Cl \\
\hline \multicolumn{4}{|c|}{ DASS-21 baseline } \\
\hline Depression & 13.0(13.5) 7.9-18.0 & $15.9(13.5) 10.8-20.9$ & $10.8(11.3) 6.6-15.0$ \\
\hline Anxiety & $11.6(12.6) 6.9-16.3$ & $14.0(13.0) 9.1-18.9$ & $9.7(10.4) 5.8-13.6$ \\
\hline Stress & $17.5(14.4) 12.2-22.9$ & $18.9(13.6)$ 13.9-24.0 & $14.2(12.0) 9.7-18.7$ \\
\hline Total & $42.1(39.0) 27.6-56.7$ & $48.8(37.7) 34.7-62.9$ & $34.7(30.3)$ 23.3-46.0 \\
\hline \multicolumn{4}{|c|}{ DASS-21 3 months } \\
\hline Depression & $9.7(11.6) 5.3-14.0$ & $15.8(14.1) 10.5-21.1$ & $9.0(10.8) 5.0-13.0$ \\
\hline Anxiety & $8.7(9.8) 5.1-12.4$ & $14.1(14.5) 8.7-19.5$ & $7.5(8.5) 4.3-10.6$ \\
\hline Stress & $14.0(12.9) 9.2-18.8$ & $16.7(13.2) 11.8-21.7$ & $11.8(10.9) 7.7-15.9$ \\
\hline Total & $32.4(31.9)$ 20.5-44.3 & 46.7(39.9) 31.8-61.6 & $34.7(30.3)$ 23.3-46.0 \\
\hline \multicolumn{4}{|l|}{ IES-R baseline } \\
\hline Intrusion & $14.8(8.9) 11.5-18.2$ & $18.4(9.3) 15.0-21.9^{*}$ & $12.1(9.4) 8.6-15.6^{*}$ \\
\hline Avoidance & $11.8(8.8) 8.5-15.1$ & 15.4(9.1) 11.9-18.7 & $9.9(7.6) 7.1-12.8$ \\
\hline Hyperarousal & $11.3(6.6) 8.9-13.8$ & $13.3(7.5)$ 10.5-16.1 & $9.2(6.8) 6.6-11.7$ \\
\hline Total & $37.9(22.9)$ 29.4-46.5 & $47.1(23.9) 38.1-56.0^{*}$ & $31.2(22.1) 23.0-39.5^{*}$ \\
\hline \multicolumn{4}{|l|}{ IES-R 3 months } \\
\hline Intrusion & $12.7(9.1) 9.3-16.1$ & $16.0(10.8) 11.9-20.0^{*}$ & $9.8(7.5)$ 7.0-12.6* \\
\hline Avoidance & $10.7(8.4) 7.5-13.8$ & $13.7(9.7)$ 10.0-17.3* & $8.0(7.4) 5.3-10.8^{*}$ \\
\hline Hyperarousal & $9.4(7.8) 6.4-12.3$ & 11.9(8.1) 8.9-15.0 & $7.3(6.0) 5.3-9.5$ \\
\hline Total & $32.7(24.0) 23.8-41.7$ & $41.6(27.5) 31.3-51.8^{*}$ & $25.1(19.4) 17.8-32.4^{*}$ \\
\hline
\end{tabular}

These are unadjusted descriptive scores only

CBT cognitive behaviour therapy, DASS-21 Depression, Anxiety and Stress Scale 21, HL healthy lifestyle, IES-R Impact of Events Scales (Revised), SD standard deviation

${ }^{*} P<.05$

\section{Results}

Baseline group characteristics and MDD or PTSD diagnosis

Table 1 shows that between groups, the participants did not differ significantly by age, sex, BMI, marital status, education, employment, role in the MVC, days since the MVC, injury type, perceived pain intensity or previous mental health history. Table 3 shows descriptive unadjusted data for DASS-21 and IES-R measures over time. No significant differences were found between groups for baseline

Table 4 Baseline and 3-month frequencies of MDD and PTSD by group according to DSM criteria

\begin{tabular}{lllll}
\hline & Control & $\mathrm{HL}$ & $\mathrm{CBT}$ & Total \\
& $n(\%)$ & $n(\%)$ & $n(\%)$ & $n(\%)$ \\
\hline MDD at baseline & $15(50.00)$ & $18(60.00)$ & $13(43.33)$ & $46(51.11)$ \\
MDD at 3 months & $14(46.66)$ & $18(60.00)$ & $14(46.66)$ & $46(51.11)$ \\
PTSD at baseline & $2(6.66)$ & $11(36.66)$ & $4(13.33)$ & $17(18.88)$ \\
PTSD at 3 months & $8(26.66)$ & $14(46.66)$ & $9(30.00)$ & $3134.44)$ \\
\hline
\end{tabular}

CBT cognitive behaviour therapy, DSM Diagnostic and Statistical Manual of Mental Disorders, $H L$ healthy lifestyle, MDD major depressive disorder, PTSD post-traumatic stress disorder
DASS-21 and domains. For IES-R, the HL group had significantly higher levels of intrusion and total scores compared to the CBT group $(P<.05)$. Table 4 shows the frequencies of diagnosed MDD and PTSD by groups over time. There was no significant difference in baseline rates of MDD, or over time between groups $\left(\mathrm{X}_{2}^{2}=1.4 ; P>.05\right)$. There was a significant difference in baseline rates of PTSD $\left(X_{2}^{2}=9.5 ; P<.01\right.$; HL had a higher rate of PTSD). No group differences in rates of PTSD occurred post-intervention.

\section{DASS-21 and IES-R total score primary outcomes}

The primary sub-group analyses compared changes in the primary measures between groups and those meeting DSM-5 criteria for MDD (no separate sub-group PTSD analyses were conducted as all but one participant with PTSD met the criteria for MDD). For participants without MDD, Fig. 2 (DASS-21 total score) and Fig. 3 (IES-R total score) show significant reductions in distress over time for all groups as measured by DASS-21 $(F(1,79)=4.0 ; P<.05)$ and by IES-R $(F(1,79)=10.0 ; P<.01)$. For those diagnosed with MDD, Fig. 4 (DASS-21 total score) shows a non-significant interaction effect in which the $\mathrm{CBT}$ and 


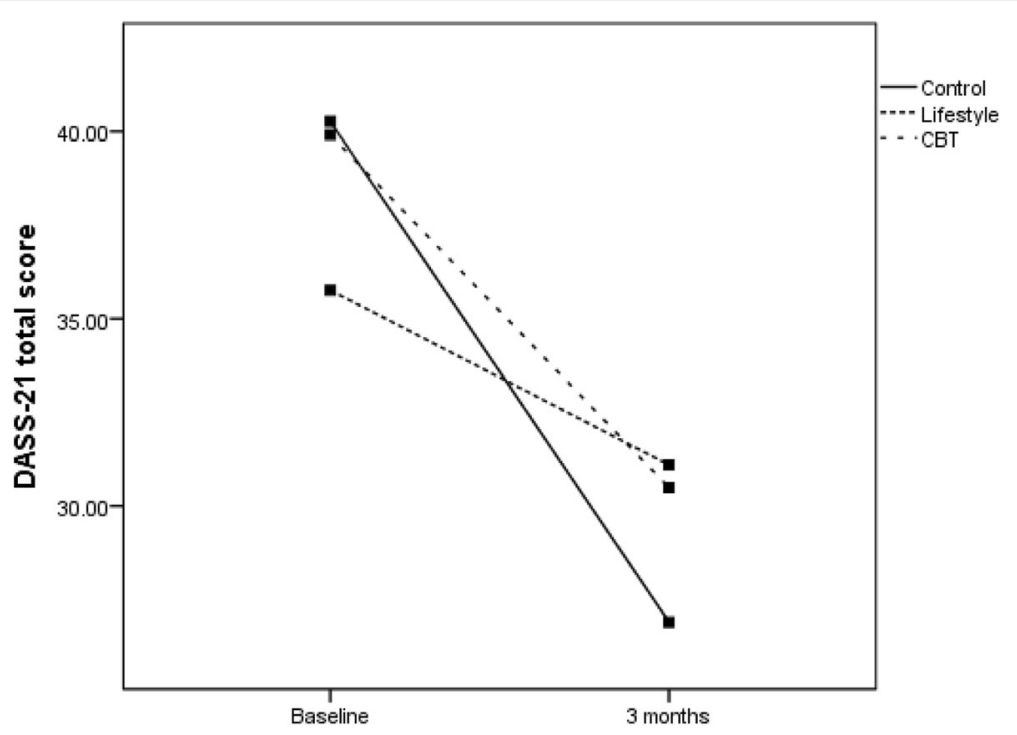

Fig. 2 DASS total score for the three groups for those without a diagnosis of MDD. CBT cognitive behaviour therapy, DASS Depression, Anxiety and Stress Scale, MDD major depressive disorder

control groups trend toward reduced distress over time compared to the HL group. For those with MDD, Fig. 5 (IES-R total score) shows a non-significant interaction effect in which the CBT group shows a trend toward reduced distress compared to the HL and control groups. Table 5 shows adjusted means for group by MDD status. Secondary analyses showed there was no main effect or interaction differences between total groups over time, though a significant reduction in distress occurred over time regardless of group for DASS-21 and IES-R $(P<.01)$.

\section{Acceptability and attrition}

Acceptability of the interventions appears to be positive. In the CBT group, 18 of 30 participants $(60.00 \%)$ were satisfied or /very satisfied with the program, 11 (36.66\%) were neither satisfied nor dissatisfied and 1 (3.33\%) was very dissatisfied. For the HL group (two had missing data), 17 of 30 participants (56.66\%) were satisfied or very satisfied, $8(26.66 \%)$ were neither satisfied nor dissatisfied and $3(10 \%)$ were dissatisfied. Similar acceptability of the CBT and HL modules was found. The participants also believed the

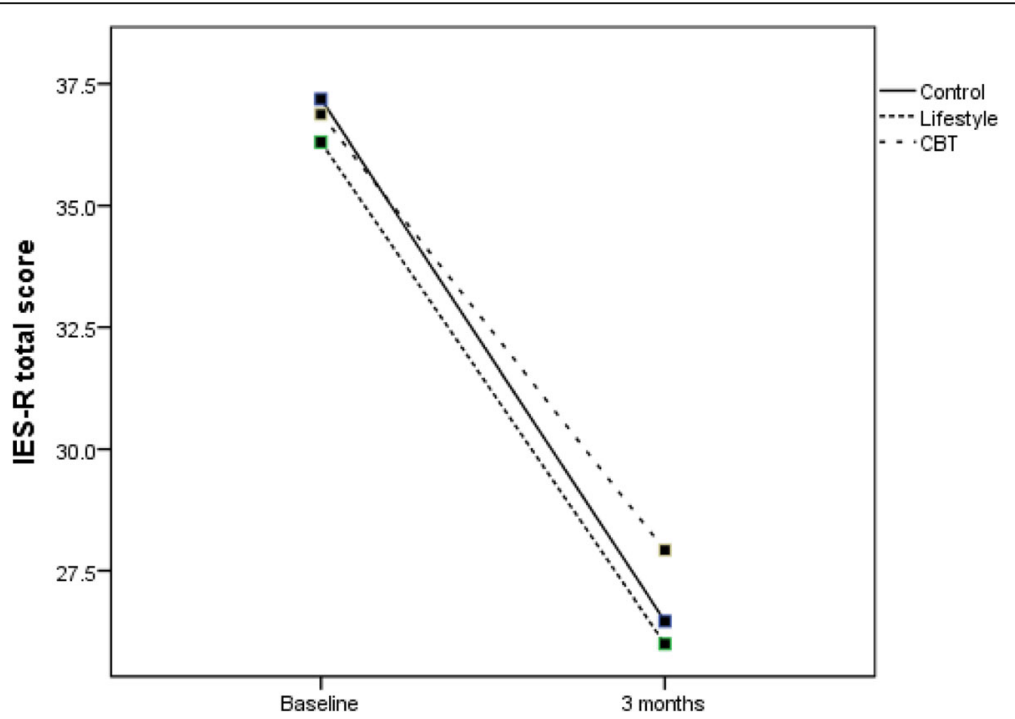

Fig. 3 IES Total for the three groups for those without diagnosis of MDD. The analysis was adjusted for baseline SF12 physical health composite index and baseline IES Intrusion domain 


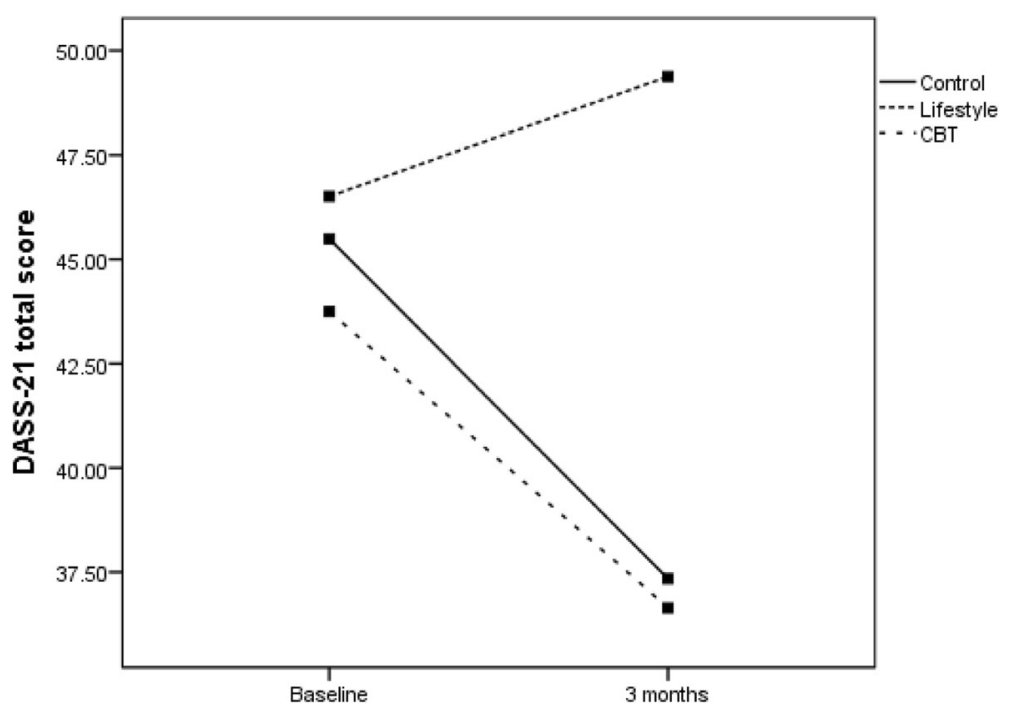

Fig. 4 DASS total for three groups for those with diagnosis of MDD. The analysis was adjusted for baseline SF12 physical health composite index and baseline DASS depression domain

program was worthwhile, with 22 of 30 (73.33\%) CBT participants and $18(60.00 \%)$ of HL participants responding positively. Finally, 21 of 30 (70.00\%) CBT participants would recommend the CBT program, whereas 20 of $30(66.66 \%)$ HL participants would recommend the HL program. Attrition rates were less than $20 \%$ (see Fig. 1).

\section{Feasibility}

While recruitment into the $\mathrm{RCT}$ has been slow, the method and goal of recruitment are feasible. At this preliminary stage, 104 participants have been recruited with
14 dropouts, resulting in 30 per group. Slow recruitment is due in part to reluctance to participate in prospective research involving treatment soon after an $\mathrm{MVC}$, especially in the context of sustaining a physical injury and being engaged in a potentially stressful compensation process. Figure 1 shows that at the time of analysis, $61 \%$ who met the inclusion criteria agreed to receive information about participation in the trial. Of those who received information, $65 \%$ consented to participate. Some participants gave a reason for non-consent: (1) no time to read modules, (2) excessive pain, (3) assistance not required and (4) legal advice against receiving assistance.

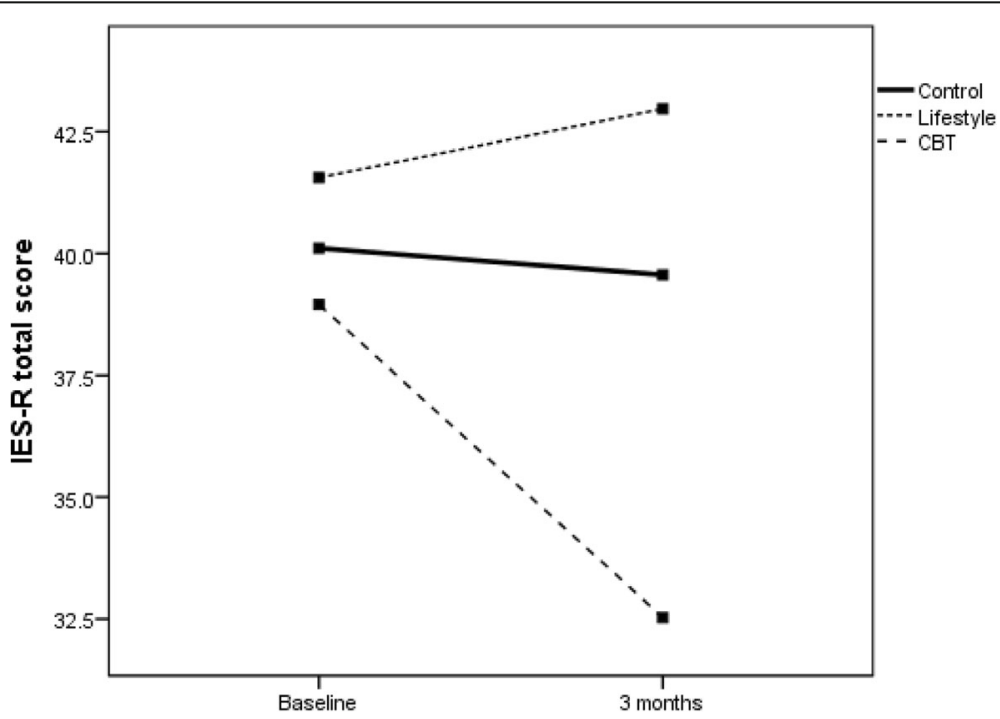

Fig. 5 IES Total for the three groups for those with a diagnosis of MDD. The analysis was adjusted for baseline SF12 physical health composite index and baseline IES Intrusion domain 
Table 5 Estimated marginal adjusted means for each group with and without MDD

\begin{tabular}{|c|c|c|c|}
\hline & Control & $\mathrm{HL}$ & CBT \\
\hline & Mean (SE) 95\% Cl & Mean (SE) 95\% Cl & Mean (SE) 95\% Cl \\
\hline \multicolumn{4}{|l|}{ DASS-21 } \\
\hline \multicolumn{4}{|l|}{ Without MDD } \\
\hline Baseline & $40.27(3.3) 33.7-46.8$ & $35.76(3.8) 28.2-43.3$ & $39.92(3.1) 33.8-46.0$ \\
\hline 3 months & $26.90(7.1) 12.7-41.1$ & $31.10((8.2) 14.8-47.4$ & $30.40(6.6) 17.4-43.6$ \\
\hline \multicolumn{4}{|l|}{ With MDD } \\
\hline Baseline & $45.49(3.2) 39.1-51.8$ & $46.51(3.1) 40.4-52.6)$ & $43.75(3.3) 37.3-50.2$ \\
\hline 3 months & $37.34(6.9) 23.7-51.0$ & $49.38(6.6) 36.3-52.5$ & $36.65(7.0) 22.7-50.7$ \\
\hline \multicolumn{4}{|l|}{ IES-R } \\
\hline \multicolumn{4}{|l|}{ Without MDD } \\
\hline Baseline & $37.18(2.2) 32.8-41.6$ & $36.31(2.5) 31.3-41.4$ & $36.87(2.1) 32.7-41.0$ \\
\hline 3 months & $26.47(4.3) 17.8-35.1$ & $26.00(5.0) 16.1-35.9$ & $27.92(4.1) 19.7-36.1$ \\
\hline \multicolumn{4}{|l|}{ With MDD } \\
\hline Baseline & $41.11(2.1) 35.9-44.3$ & $41.56(2.1) 37.3-45.8$ & $38.95(2.2) 34.5-43.4$ \\
\hline 3 months & $39.56(4.2) 31.3-47.8$ & $42.97(4.2) 34.6-51.3$ & $32.53(4.4) 23.8-41.2$ \\
\hline
\end{tabular}

Covariates for the DASS-21 analysis were DASS-21 depression and physical component summary. Covariates for the IES-R analysis were IES-R intrusion and physical component summary

CBT cognitive behaviour therapy, Cl confidence interval, DASS-21 Depression, Anxiety and Stress Scale 21, HL healthy lifestyle, IES-R Impact of Events Scales (Revised), MDD major depressive disorder SE standard error of the mean

These reflect the difficulty of recruiting through an insurer, and further, delays occurred due to frequent re-organisations of the insurance company and the limited time for case managers to introduce the research to potential participants. Recruitment within 4 months from MVC to claim lodgement proved problematic due to: (1) delays in achieving contact with participants and (2) the need for frequent reminders to provide consent and complete assessments. Table 1 shows that there were approximately 3 months (mean days $=82.09$, standard deviation, $\mathrm{SD}=57.08$ ) from the MVC to completion of the baseline assessment. Completion of the post-intervention assessment often did not occur immediately after program completion, often taking several weeks and repeated phone calls (mean calls $=2.99, \mathrm{SD}=2.4$ ). Further, the need for frequent reminders to complete assessments may have negatively influenced participants' program feedback compared to their positive verbal feedback received during the intervention. Rapport ratings (Table 1) suggest more positive rapport was established with the CBT group than the HL group, with 28 of 30 (93.33\%) positive ratings for CBT compared to 23 of $30(76.67 \%)$ positive ratings for the HL group.

Finally, not all who met the inclusion criteria had access to email, resulting in some postal delivery of modules. Table 1 shows email was an efficient means of delivery with $84(93.33 \%)$ of 90 participants receiving their modules by email, and only 6 (6.67\%) having no access to email who received modules via the post.

\section{Discussion}

The preliminary results demonstrate that brief email-delivered CBT or HL interventions with clinically focussed telephone support is most likely not required for participants without a diagnosis of MDD or PTSD, at least up to 3 months post-MVC. This conclusion is supported by the significantly reduced psychological distress over time regardless of group, shown in Figs. 2 and 3. For those diagnosed with MDD soon after their MVC, the findings shown in Fig. 5 offer rudimentary support for the efficacy of a brief CBT intervention delivered by email to reduce psychological distress as assessed by the IES-R. Nevertheless, Fig. 4 shows DASS-21 total results by group, suggesting benefit can be gained by either CBT or regularly calling people following an MVC who were engaged in seeking compensation and providing them with compensation-related reading material. In contrast, the benefits of providing a brief HL intervention for those with MDD are not promising and potentially harmful, given the results in Figs. 4 and 5 indicate that these participants did not show reduced distress over time. Whilst there was no change in MDD or PTSD diagnoses by 3 months, the major aim of reducing psychological distress appears to have been achieved for those without MDD, with positive signs of reduced distress in those with MDD in the CBT and control groups.

These preliminary findings are important, first because they have influenced the decision to cease recruitment to the HL intervention. Further, they provide possible 
direction for managing psychological distress in people sustaining MVC-related injuries who are engaged in seeking compensation; a situation identified as having a significant risk of elevated psychological distress and adverse outcomes, such as a delayed return to work [8], as well as lengthier and more costly claims [43]. With completion of the trial, further analysis of the total participant pool with 6- and 12-month post-MVC longitudinal data will help clarify the efficacy of the brief interventions. This is especially true for determining the usefulness of HL interventions for those diagnosed with MDD (or PTSD) soon after their MVC. For example, physically injured MVC survivors may be overwhelmed or irritated by an intervention that requests they monitor and improve their diet, sleep and exercise behaviour soon after a traumatic event, strategies that may be perceived to be unrelated to their injuries. Accordingly, the $\mathrm{RCT}$ will now continue as a two-arm trial, with the CBT and control groups.

Preliminary acceptability and feasibility results including adherence rates, delivery methodology and researcher-rated rapport all appear positive. However, the slow recruitment into the trial through the insurer partners has challenged an initial goal of the study, which was to recruit as early as possible after the MVC to help prevent the development of MDD and PTSD $[11,30]$. On completion of the study, we intend to investigate whether those recruited earlier after the MVC (e.g. within 2 months) have superior outcomes than those recruited later (e.g. 3-4 months post-MVC).

\section{Study limitations}

The 104 (30/30/30 with attrition) participants are more than likely a biased sample, given that fewer than $50 \%$ of the participants approached by the insurers agreed to enter the trial. Reasons for this include approaching participants at a stressful time, a perception of no need for psychological help and the opt-in style used for recruitment. It is also noted that the sample appears highly educated and the impact of all these limitations on bias in the sample needs to be considered when drawing conclusions. A further limitation relates to pre-morbid mental health history. This information is not routinely collected by insurance companies. However, the information collected about prior treatment by a psychologist or psychiatrist and on whether participants are taking prescribed psychiatric medications do provide a proxy for pre-morbid mental health history, which has been shown to be a strong predictor of post-MVC psychological distress $[44,45]$. However, Table 1 showed pre-morbid psychological distress was not significantly different between the groups. We intend to explore fully the relationship between pre-morbid psychological distress and distress outcomes at the completion of the trial.

\section{Conclusions}

This is the first study to investigate a brief email-delivered clinically focussed treatment offered to consenting MVC survivors irrespective of crash fault status or pre-morbid physical and mental health. Preliminary findings have identified the need to discontinue the HL intervention. The findings also offer preliminary guidance on improving mental health status for those with an MVC-related injury and engaged in seeking compensation. The limited compensation and health resources need to be directed to where they are needed most to ensure timely and effective recovery. Moreover, there is need to improve the cost-effectiveness of claims managed by insurance companies. For example, for those assessed as not meeting psychiatric criteria for MDD or PTSD, a beneficial long-term outcome may require only the provision of relevant claim material and a fortnightly telephone call, 36 months after seeking compensation. Further, for those identified with MDD, a cautious interpretation may be that the provision of brief CBT sessions early after an MVC may protect against chronic psychological disorders. The preliminary data suggest the RCT is feasible and acceptable and therefore, the study will continue to recruit and conduct follow-up assessments to 12 months post-MVC. More detailed analysis of the acceptability of the interventions and their potential associations with outcomes will be reported in future publications at the conclusion of the RCT.

\section{Additional file}

Additional file 1: Consort checklist: recommended items to address in a clinical trial. (DOC $217 \mathrm{~kb}$ )

\section{Abbreviations \\ BMI: Body mass index; CBT: Cognitive behaviour therapy; Cl: Confidence interval; DASS-21: Depression, Anxiety and Stress Scale 21; DSM-5: Diagnostic and Statistical Manual of Mental Disorders, 5th Edition; HL: Healthy lifestyle; IES-R: Impact of Events Scales (Revised); MANCOVA: Multi-variate analysis of covariance; MDD: Major depressive disorder; MVC: Motor vehicle crash; NSW: New South Wales; PTSD: Post-traumatic stress disorder; RCT: Randomised controlled trial; SD: Standard deviation; SE: Standard error of the mean; SF: Short Form}

\section{Acknowledgements}

The authors acknowledge the contributions of the three insurance companies, Suncorp, National Roads and Motorists' Association and

Transport Accident Commission. We also acknowledge all participants, without whom this study would not be possible.

Funding

This research is being funded by the State Insurance Regulatory Authority, grant MAA 14/366. The funding source had no role in the design, collection, analysis, interpretation or writing up of the study. 


\section{Availability of data and materials}

Interventions and measures described in the manuscript are available to any scientist wishing to use them without breach of participant confidentiality. The raw data will not be shared, as they will not be reported or published at any time as individual data, and because the multi-site insurance companies have provided claimant contact details strictly for only the RCT.

\section{Authors' contributions}

All authors were responsible for study conception and design and the trial registration. YT was responsible for data analysis. $R G, A C, B G$ and IC were the principal investigators and responsible for implementation of the study, and the writing of publications. All authors critically reviewed, read and approved the final manuscript

\section{Ethics approval and consent to participate}

This research received approval from each site and was fully compliant with the code of ethics of the World Medical Association (Declaration of Helsinki), as well as the standards established by the authors' institutional review board and the granting agency. Written informed consent has been sought from all participants prior to participation. The Human Research Ethics Committee granted approval on 26 February 2015 (number 2015/016), as did the multi-site insurance companies, and the trial was registered on 9 April 2015 (registration number ANZCTR - ACTRN12615000326594).

\section{Consent for publication}

Written informed consent has been obtained from the participants for publication of the manuscript's grouped data and figures (data relating to any single individual will not be published). The completed consent forms are securely held by the authors' institution and are available for review by the editor-in-chief

\section{Competing interests}

The authors declare that they have no competing interests.

\section{Publisher's Note}

Springer Nature remains neutral with regard to jurisdictional claims in published maps and institutional affiliations.

Received: 17 November 2017 Accepted: 1 June 2018 Published online: 27 June 2018

\section{References}

1. Bryant RA, O'Donnell ML, Creamer M, McFarlane AC, Clark CR, Silove D. The psychiatric sequelae of traumatic injury. Am J Psychiatry. 2010;167(3):312-20.

2. Bryant RA, O'Donnell ML, Creamer M, McFarlane AC, Silove D. A multisite analysis of the fluctuating course of posttraumatic stress disorder. JAMA Psychiatry. 2013;70(8):839-46.

3. Hodgson RC, Webster RA. Mediating role of peritraumatic dissociation and depression on post-MVA distress: path analysis. Depress Anxiety. 2011;28(3):218-26.

4. Nickerson A, Aderka IM, Bryant RA, Hofmann SG. The role of attribution of trauma responsibility in posttraumatic stress disorder following motor vehicle accidents. Depress Anxiety. 2013;30(5):483-8.

5. Craig A, Tran Y, Guest R, Gopinath B, Jagnoor J, Bryant RA, et al. Psychological impact of injuries sustained in motor vehicle crashes: systematic review and meta-analysis. BMJ Open. 2016;6(9):e011993.

6. Gopinath B, Harris IA, Nicholas M, Casey P, Blyth F, Maher CG, et al. A comparison of health outcomes in older versus younger adults following a road traffic crash injury: a cohort study. PLoS One. 2015:10(4):e0122732.

7. Craig A, Tran Y, Cameron ID. Psychopathology associated with motor vehicle crashes. Psychopathology: theory, perspectives and future approaches. Hauppauge: Nova Biomedical Books; 2013. p. 323-42.

8. Elbers NA, Hulst L, Cuijpers P, Akkermans AJ, Bruinvels DJ. Do compensation processes impair mental health? A meta-analysis. Injury. 2013;44(5):674-83.

9. Papadakaki M, Ferraro OE, Orsi C, Otte D, Tzamalouka G, Von-der-Geest M, et al. Psychological distress and physical disability in patients sustaining severe injuries in road traffic crashes: results from a one-year cohort study from three European countries. Injury. 2017:48(2):297-306.

10. Schaafsma F, De Wolf A, Kayaian A, Cameron ID. Changing insurance company claims handling processes improves some outcomes for people injured in road traffic crashes. BMC Public Health. 2012;12(1):36.
11. Guest R, Tran Y, Gopinath B, Cameron ID, Craig A. Psychological distress following a motor vehicle crash: a systematic review of preventative interventions. Injury. 2016;47(11):2415-23.

12. Kearns MC, Ressler KJ, Zatzick D, Rothbaum BO. Early interventions for PTSD: a review. Depress Anxiety. 2012;29(10):833-42.

13. Rohrer JE, Pierce JR, Blackburn C. Lifestyle and mental health. Prev Med. 2005;40(4):438-43.

14. Brouwer W, Kroeze W, Crutzen R, de Nooijer J, de Vries NK, Brug J, et al. Which intervention characteristics are related to more exposure to internetdelivered healthy lifestyle promotion interventions? A systematic review. J Med Internet Res. 2011;13(1):e2.

15. Rowley KG, Daniel M, Skinner K, Skinner M, White GA, O'Dea K. Effectiveness of a community-directed 'healthy lifestyle'program in a remote Australian aboriginal community. Aust N Z J Public Health. 2000:24(2):136-44

16. Bains MK, Scott S, Kellett S, Saxon D. Group psychoeducative cognitivebehaviour therapy for mixed anxiety and depression with older adults. Aging Ment Health. 2014;18(8):1057-65.

17. Craig A. Resilience in people with physical disabilities. In: Kennardy P, editor. The Oxford handbook of rehabilitation psychology. New York: Oxford University Press; 2012. p. 474-91.

18. Hedman E, Ljótsson B, Lindefors N. Cognitive behavior therapy via the internet: a systematic review of applications, clinical efficacy and costeffectiveness. Expert Rev Pharmacoecon Outcomes Res. 2012;12(6):745-64.

19. Morley S, Eccleston C, Williams A. Systematic review and meta-analysis of randomized controlled trials of cognitive behaviour therapy and behaviour therapy for chronic pain in adults, excluding headache. Pain. 1999:80(1):1-13.

20. Hancock K, Craig A. The influence of a healthy lifestyle program in a work environment: a controlled long term study. J Occup Health Safety Aust N Z. 1996;12(2):193.

21. Cooney G, Dwan K, Greig C, Lawlor D, Rimer J, Waugh F, Mead GE, et al. Exercise for depression. Cochrane Database Syst Rev. 2013;9(9):CD 004366.

22. Craig A, Elbers N, Jagnoor J, Gopinath B, Kifley A, Dinh $M$, et al. The psychological impact of traffic injuries sustained in a road crash by bicyclists: a prospective study. Traffic Injury Prev. 2017;18(3):273-80.

23. Hamer M, Steptoe A. Association between physical fitness, parasympathetic control, and proinflammatory responses to mental stress. Psychosom Med. 2007:69(7):660-6.

24. Strine TW, Chapman DP. Associations of frequent sleep insufficiency with health-related quality of life and health behaviors. Sleep Med. 2005;6(1):23-7.

25. Ströhle A. Physical activity, exercise, depression and anxiety disorders. J Neural Transm. 2009;116(6):777-84.

26. Andersson G, Cuijpers P. Internet-based and other computerized psychological treatments for adult depression: a meta-analysis. Cogn Behav Ther. 2009:38(4):196-205.

27. Lewis CE, Farewell D, Groves V, Kitchiner NJ, Roberts NP, Vick T, et al. Internet-based guided self-help for posttraumatic stress disorder (PTSD): randomized controlled trial. Depress Anxiety. 2017:34(6):555-65.

28. Gilbody S, Littlewood E, Hewitt C, Brierley G, Tharmanathan P, Araya R, et al. Computerised cognitive behaviour therapy (CCBT) as treatment for depression in primary care (REEACT trial): large scale pragmatic randomised controlled trial. BMJ. 2015:351:h5627.

29. Knowles SE, Lovell K, Bower P, Gilbody S, Littlewood E, Lester H. Patient experience of computerised therapy for depression in primary care. BMJ Open. 2015;5(11):e008581.

30. Guest R, Tran Y, Gopinath B, Cameron ID, Craig A. Prevention of the development of psychological distress following a motor vehicle crash: study protocol for a randomized controlled trial. Trials. 2016;17(1):317.

31. Harris IA, Young JM, Jalaludin BB, Solomon MJ. The effect of compensation on general health in patients sustaining fractures in motor vehicle trauma. J Orthop Trauma. 2008:22(4):216-20.

32. Henry JD, Crawford JR. The short-form version of the depression anxiety stress scales (DASS-21): construct validity and normative data in a large nonclinical sample. Br J Clin Psychol. 2005;44(2):227-39.

33. Lovibond PF, Lovibond SH. The structure of negative emotional states: comparison of the depression anxiety stress scales (DASS) with the Beck depression and anxiety inventories. Behav Res Ther. 1995;33(3): 335-43.

34. Weiss D, Marmar C, Wilson J, Keane T. Assessing psychological trauma and PTSD. The Impact of Events Scale-Revised; 1997. p. 399-411. 
35. Beck JG, Grant DM, Read JP, Clapp JD, Coffey SF, Miller LM, et al. The impact of event scale-revised: psychometric properties in a sample of motor vehicle accident survivors. J Anxiety Disord. 2008;22(2):187-98.

36. American Psychiatric Association. Diagnostic and statistical manual of mental disorders (DSM-5). Arlington: American Psychiatric Publishing; 2013.

37. Ware JE Jr, Kosinski M, Keller SD. A 12-item short-form health survey: construction of scales and preliminary tests of reliability and validity. Med Care. 1996;34(3):220-33.

38. Dear BF, Titov N, Perry KN, Johnston L, Wootton BM, Terides MD, et al. The pain course: a randomised controlled trial of a clinician-guided internetdelivered cognitive behaviour therapy program for managing chronic pain and emotional well-being. Pain. 2013;154(6):942-50.

39. Titov N, Dear BF, Johnston L, Lorian C, Zou J, Wootton B, et al. Improving adherence and clinical outcomes in self-guided internet treatment for anxiety and depression: randomised controlled trial. PLoS One. 2013:8(7):e62873.

40. IBM Analytics. IBM SPSS Software 2017 [Available from: http://www.ibm. com/analytics/au/en/technology/spss/. Accessed 13 June 2018.

41. Committee for Medicinal Products for Human Use. Guideline on adjustment for baseline covariates in clinical trials 2015 [Available from: http://www. ema.europa.eu/docs/en_GB/document_library/Scientific_guideline/2015/03/ WC500184923.pdf. Accessed 13 June 2018.

42. de Boer MR, Waterlander WE, Kuijper LD, Steenhuis IH, Twisk JW. Testing for baseline differences in randomized controlled trials: an unhealthy research behavior that is hard to eradicate. Int J Behav Nutr Phys Act. 2015;12(1):4.

43. Guest R, Tran Y, Gopinath B, Cameron ID, Craig A. Psychological distress following a motor vehicle crash: evidence from a state-wide retrospective study examining settlement times and costs of compensatjion claims. BMJ Open. 2017;e017515.

44. Craig A, Nicholson Perry K, Guest R, Tran Y, Dezarnaulds A, Hales A, et al. Prospective study of the occurrence of psychological disorders and comorbidities after spinal cord injury. Arch Phys Med Rehab. 2015;96(8):1426-34.

45. Sareen J, Erickson J, Medved MI, Asmundson GJG, Enns MW, Stein M, et al. Risk factors for post-injury mental health problems. Depress Anxiety. 2013;30(4):321-7.

\section{Ready to submit your research? Choose BMC and benefit from:}

- fast, convenient online submission

- thorough peer review by experienced researchers in your field

- rapid publication on acceptance

- support for research data, including large and complex data types

- gold Open Access which fosters wider collaboration and increased citations - maximum visibility for your research: over $100 \mathrm{M}$ website views per year

At BMC, research is always in progress.

Learn more biomedcentral.com/submissions 